\title{
Nanocubes of Palladium, Simple, Green Approach and Catalytic Properties Under Continuous Hydrogenation System
}

\author{
B. G. Bharate* \\ Department of Chemical and Biomolecular Engineering, Sogang University, Seoul (Sinsu-dong), Republic of Korea
}

*Corresponding author: Bapurao G Bharate, Department of Chemical and Biomolecular Engineering, 35 Baekbeom-ro (Sinsudong), Mapo-gu, Republic of Korea

\begin{tabular}{|c|c|}
\hline ARTICLE INFO & ABSTRACT \\
\hline Received: 觜 July 16, 2019 & \multirow{4}{*}{$\begin{array}{l}\text { Nanocubes of palladium has been synthesized by refluxing palladium chloride in } \\
\text { ethanol. A gram level of palladium nanoparticles was synthesized in } 1.5 \text { hours. Ethanol } \\
\text { acts as a reducing agent as well as the medium of reaction in the presence of ethylene } \\
\text { glycol it forms nanocubes while in absence it form spherical particles with pronounced } \\
\text { agglomeration. The reduction process has been monitored by UV-Visible spectroscopy } \\
\text { which revealed disappearance of band at } 421 \mathrm{~nm} \text { due to } \mathrm{PdCl}_{2} \text {. X-ray analysis discloses the } \\
\text { face centered cubic crystal structure. TEM micrograph confirms the particle size is about } \\
10 \mathrm{~nm} \text { with cubic shape. The catalytic activity of as synthesized palladium nanoparticles } \\
\text { was tested using H-Cube flow hydrogenation system and it was found that palladium } \\
\text { nanoparticles are active catalyst for the reduction of nitro to amine group. }\end{array}$} \\
\hline Published: 㗀 July 23, 2019 & \\
\hline $\begin{array}{l}\text { itation: B. G. Bharate. Nanocubes of } \\
\text { alladium, Simple, Green Approach and } \\
\text { Catalytic Properties Under Continuous }\end{array}$ & \\
\hline $\begin{array}{l}\text { Hydrogenation System. Biomed J } \\
\text { Sci \& Tech Res 19(5)-2019. BJSTR. } \\
\text { MS.ID.003375. }\end{array}$ & \\
\hline
\end{tabular}

\section{Introduction}

There are large number of reports on the synthesis of palladium nanoparticles due to several important applications. There is much interest on nanostructured transitional metal particles, because of their characteristic properties, strikingly different from the bulk. Nanoparticles hold promise to employ them as innovative materials with new electronic, magnetic and thermal properties. They have a large surface-to-volume ratio and consequently exhibit increased surface activity compared to bulk material, which is important parameter for a good catalyst [1-5]. The reduction of aromatic nitro compounds to amines is an important process because they are useful precursors and intermediates in the preparation of dyes, polymers, pharmaceutical substances, agricultural chemicals and other products. There are number of methods for the reduction of nitro group, but the poor selective reduction of the nitro to the amino group in the presence of other reducible functionalities can make this reaction a challenging task. The surfactant or capping agent plays an important role because it can reduce the catalytic activity of the nanoparticles by hindering substrate contact or by blocking product departure from the surface. Thus, there is a growing need for the development of new methods for the synthesis of well-dispersed nanoparticles and stabilizers which do not affect the catalytic activity [6-10].

Thus, nanosize transition-metal particles have attracted attention from both fundamental and technological viewpoints because of quantum size effect. Among all the transition metals, palladium is of interest because of its high catalytic activity in several reactions, e.g. hydrogenation, Heck reaction, C-C bond formation reaction, Suzuki reaction, cyclization reaction, etc. Several reports have been of palladium nanoparticles in catalysis, electronics, published regarding the preparation of stable palladium nanoparticles. Among them are the sonochemical method, electrochemical method, microemulsion technique and other methods. [11-16] However, the reproducible preparation of small, stable palladium nanoparticles with tight size distribution is of immense importance and still remains a challenging task. We herein present a simple one-step preparation method for the preparation of palladium nanoparticles by use of ethanol and ethylene glycol as surfactant as well as reducing agent without using any external reducing agent and its catalytic properties using Thales nano H-Cube flow continuous hydrogenation system. 


\section{Experimental}

\section{Materials and General Methods}

Palladium chloride purchased from Sigma Aldrich and used as received. Ethanol and ethylene glycol used are of A.R grade from local vender. The Nanopwder formed were characterized by following Technique. UV-Visible spectra were measured on JASCO V-570 spectrophotometer. Scanning electron microscopy (SEM) was Recorded on Philips XL-30. Powder X-ray diffraction (XRD) patterns were obtained using Miniflex Rigaku model. TEM was performed on Tecnai-G $\mathrm{G}^{2}$. Palladium nanoparticles was synthesized by modified procedure [17] with high yield and good quality. In which Palladium chloride 0.25 and $0.5 \mathrm{gm}$ is refluxed in a sufficient Quantity of ethanol and $2 \mathrm{ml}$ of ethylene glycol in a round bottom flask under $\mathrm{N}_{2}$ atmosphere for about 2 hours. The reaction progress was monitored by UV- Visible spectroscopy which revealed disappearance of band at $421 \mathrm{~nm}$ due to $\mathrm{PdCl}_{2}$. After the completion of the reaction, the centrifugation of the reaction mixture yielded black powder of palladium nanoparticles in quantitative yield. The yield of palladium nano powder is about $91 \%$ which is quite good.

\section{Results and Discussion}

\section{Synthesis and Characterization}

Gram level preparation of Palladium nanoparticles has been obtained by the use of ethanol and small quantity of ethylene glycol as a surfactant as well as reducing agent in good quality and quantity. The reduction of metal salts leads to formation of species with lower oxidation states. Reduction can be accomplished by use of common reagents such as sodium borohydride hydrate, hydrazine hydrate, sodium formaldehyde Sulphoxylate and many others. The major difficulty however has always been that the instant reduction of such salts leads to non-stoichiometry in the end product and their uncontrolled shapes and sizes. Such preparations often yield nanoparticles with defects due to the instant reduction of the salts. The scheme for the formation Palladium nanoparticles from the reduction of palladium chloride is proposed in Figure 1. In the present study it has been observed that the reduction of palladium chloride with ethanol yield spherical particles with prolonged agglomeration and do not show the activity toword the reduction of nitro to amine group. However reduction of palladium chloride with ethanol in presence of ethylene glycol yield cubic particles without prolonged agglomeration and show the catalytic activity toward the reduction of nitro to amine group in presence of $\mathrm{H}$-Cube flow continuous hydrogenation system, The initial formation of palladium nanoparticles was examined by absorption spectroscopy.

\section{$\mathrm{PdCl}_{2}+\mathrm{C}_{2} \mathrm{H}_{5} \mathrm{OH}$



Figure 1: Scheme for the formation of Palladium nanoparticles.
The UV-Visible spectrum of the palladium chloride shows an absorption maxima at $421 \mathrm{~nm}$ which slowly disappeared during the reaction and finally the spectrum showed a straight line indicating completion of reduction process Figure 2. The centrifugation of the reaction mixture yielded highly stable black powder of palladium nanoparticles in quantitative yield. The yield of palladium nano powder is about $91 \%$ which is quite good. The X-ray powder diffraction pattern confirmed the face centered cubic structure of Palladium nanoparticles which shows the reflection correspond to $111,200,220,311$ and 222 crystal planes. XRD pattern showed reflections at $2 \theta ; 40,46,68,82$ and 87 o correspond to phase pure palladium nano-particles. The interplanar spacing (dhkl) values $2.24,1.94,1.37$ and $1.17 \AA$ ) and the lattice constant (3.88 ̊) calculated from the XRD spectrum of palladium nanoparticles are in agreement with the standard palladium values (JCPDS card 461043). XRD pattern of as synthesized palladium nanoparticles shows sharper peak which hints the crystalline nature of palladium nanoparticles [18]. The particle size of the as synthesized Palladium nanoparticles was calculated from its XRD patterns using Debye-Scherrer (about 8nm) which is well well matches with TEM Figure 3.

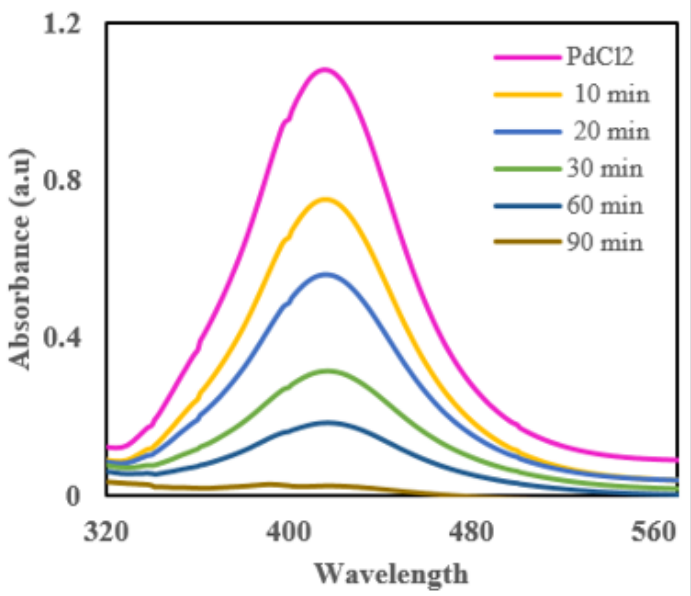

Figure 2: UV-Visible spectra during formation of Pd nanoparticles
a) $\quad \mathrm{PdCl}_{2}$
b) $10,20,30,60$ and $90 \mathrm{~min}$.

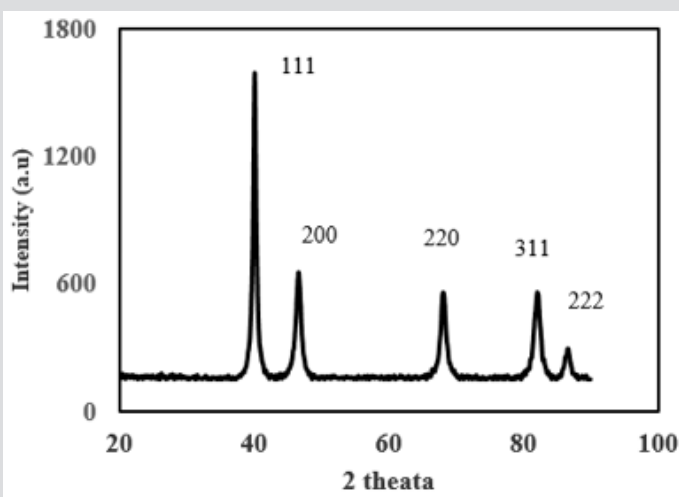

Figure 3: XRD of as-prepared palladium nanoparticles. 
The TEM micrograph of Palladium nanoparticles is shown in Figure 4. The micrograph revealed that well dispersed nanoparticles of about $10 \mathrm{~nm}$ diameter is formed. TEM images confirm cubic shape of nanoparticles. TEM images also shows well defined fringed indicating crystalline nature of nanoparticles. The electron diffraction patterns recorded for palladium nanoparticles showed all relevant concentric rings for various crystal planes for fcc crystal structure of the metal. The SEM micrograph of palladium powder also shows cubic morphology for as-prepared powders without pronounced agglomeration. The micrograph hint that the small particles are quite scattered indicating high volume to surface area Figure 5. Particle size analysis of as synthesized palladium nanoparticles was carried out by using dynamic light scattering techniques (DLS). DLS study shows that narrow size distribution within the range of $\sim 10 \mathrm{~nm}$ was observed. Which are well matches with TEM and XRD measurement.


Figure 4: TEM images of as-prepared Palladium nanoparticles.

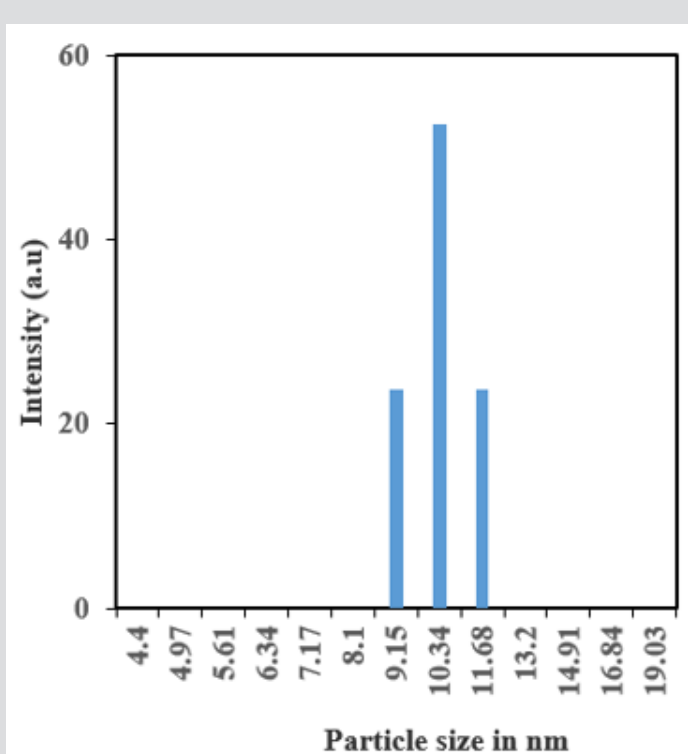

Figure 5: Particle size distribution of as prepared Palladium Nanoparticles

\section{Catalysis Properties}

Catalysis properties of the as synthesized palladium nanoparticles have been performed on the $\mathrm{H}$-Cube flow hydrogenation system. The H-Cube reactor was used for reduction of nitro group under hydrogenation flow conditions. All catalytic reactions were performed using $\mathrm{H}$-Cube $\mathrm{Pro}^{\mathrm{TM}}$. Using the $\mathrm{H}$-Cube continuous flow reactor, as synthesized palladium nanoparticles was filled into the catalyst cartridge removed the air gapes and cartridge is sealed. Starting with a control experiment of hydrogenation of nitrobenzene, 4-nitrotolulene with as synthesized palladium nanoparticles. The reactions run at a $0.01 \mathrm{M}$ concentration and nearly $100 \% \mathrm{H}_{2}$ production. Reaction conditions: Solvent: Ethanol, pressure: 1bar, substrate concentration $0.01 \mathrm{~mol} / \mathrm{dm}^{3}$, temperature: $40^{\circ} \mathrm{C}$, flow rate: $0.5 \mathrm{~mL} / \mathrm{min} .0 .50 \mathrm{~g}$ nitrobenzene or $0.5 \mathrm{~g} 4$-nitrotolulene in ethanol was continuously pumped though the catalyst bed at $0.5 \mathrm{~mL} /$ min at $40^{\circ} \mathrm{C}$ reaction temperature resulting in $94 \%$ of yield of final product within $60 \mathrm{~min}$. The solution of starting material and in-situ generated high pressure hydrogen was reacted inside the Cat Cart at atmospheric pressure. 
The conversions of the nitro to amine group were monitored by TLC. The reaction can be conveniently monitored by TLC, following the disappearance of 4-nitrobenzene and 4-nitrotolene spot and appearing new spot for the formation of amine. In order to confirm the TLC analysis, we performed mass spectrometry experiments. Our mass spectrometry results are consistent with the formation of amino products appearing molecular ion peak at $\mathrm{m} / \mathrm{z} 93$ and 107 for aniline and 4-amino toluene respectively. Further the amine product is analysed by IR spectroscopy which shows appearance new band at $3200 \mathrm{~cm}^{-1}$ due to amine group which not present in spectrum of nitro compound. The conditions described above give $94 \%$ conversion to product in one flow through the system. The experiments showed that the Pd nanoparticles, which are a green alternative to the bulk metal catalyst, is active under flow conditions. Experimental results show that as synthesized palladium nanoparticles have high activity at low temperatures for the reduction of nitro to amine group.

\section{Conclusion}

In conclusion, Nanocubes of palladium nanoparticles are synthesized up to few gram level in good the yield by green approach. XRD pattern showed that phase pure palladium nanoparticles is formed. TEM study showed that the particle size of palladium nanoparticles is about $10 \mathrm{~nm}$. Cubic morphology of the particles is established by SEM. These result shows that it is possible to prepare palladium nanoparticles with good yield by easy and green process for catalytic activity. The Palladium catalysed amine forming reaction of nitrobenzene or 4-nitrotolulene have been carried out and it was found that as synthesized palladium catalyst worked as active catalyst for the reduction of nitro group. The above results shows the usefulness of flow chemistry in the testing of as synthesized palladium nanoparticles in the reduction of nitro group to amine with fast reactions, safe high pressure hydrogen generation from water, catalyst cartridges, and use under mild conditions.

\section{References}

1. J Cookson (2012) The Preparation of Palladium Nanoparticles Controlled particle sizes are key to producing more effective and efficient materials. Platinum Metals Rev 56(2): 83-98.

2. S Link, MA El Sayed (1999) Spectral Properties and Relaxation Dynamics of Surface Plasmon Electronic Oscillations in Gold and Silver Nanodots and Nanorods. J Phys Chem B 103(40): 8410-8426.

\section{ISSN: 2574-1241}

DOI: 10.26717/BJSTR.2019.19.003375

B. G. Bharate. Biomed J Sci \& Tech Res

This work is licensed under Creative Commons Attribution 4.0 License

Submission Link: https://biomedres.us/submit-manuscript.php
3. H Chen, G Wei, A Ispas, SG Hickey, L Eychmüller, et al. (2010) Synthesis of Palladium Nanoparticles and Their Applications for Surface-Enhanced Raman Scattering and Electrocatalysis.J Phys Chem C 114(50): 2197621981.

4. SW Kim, J Park, Y Jang, Y Chung, S Hwang, et al. (2003) Synthesis of Monodisperse Palladium Nanoparticles Nano Letters 3(9): 1289-1291.

5. T Sun, Z Zhang, J Xiao, C Chen, F Xiao, et al. (2013) Facile and green synthesis of palladium nanoparticles-graphene-carbon nanotube material with high catalytic activity. Sci Rep 3: 1-6.

6. F Souza, H Fiedler, F Nome (2016) Zwitterionic Surfactant Stabilized Palladium Nanoparticles as Catalysts in Aromatic Nitro Compound Reductions. J Braz Chem Soc 27(2): 372-381.

7. P Sangeeta, K Shanthi, KS Rama Rao, B Viswanathan, P Selvam, et al. (2009) Appl Catal A 353: 160-165.

8. A Kumari, M Venkatesham, D Ayodhya, G Veerabhadra (2015) Applied Nanoscience. Appl Nanosci 5: 315-320.

9. YM Lu, HZ Zhu, WG Li, B Hu, SH Yu, et al. (2013) Size-controllable palladium nanoparticles immobilized on carbon nanospheres for nitroaromatic hydrogenation. J Mater Chem A 1: 3783-3788.

10. JJ Griebel, MD Gawryla, HW Milliman, DA Schiraldi (2016) Clay Aerogel Supported Palladium Nanoparticles as Catalysts. Gels 2(2): 15: 1-8.

11. RB Nasirbaig, RS Varma (2014) Magnetic Carbon-Supported Palladium Nanoparticles: An Efficient and Sustainable Catalyst for Hydrogenation Reactions. ACS Sustainable Chem Eng 2(9): 2155-2158.

12. OM Wilson, MR Knecht, JC Garcia Martinez, RM Crook (2006) Effect of $\mathrm{Pd}$ nanoparticle size on the catalytic hydrogenation of allyl alcohol. J Am Chem Soc 128(14): 4510-4511.

13. NR Jana, ZL Wang, T Pal (2000) Redox Catalytic Properties of Palladium Nanoparticles: Surfactant and Electron Donor-Acceptor Effects. Langmuir 16(6): 2457-2463.

14.Y Niu, LK Yeung, RM Crook (2001) Size-Selective Hydrogenation of Olefins by Dendrimer-Encapsulated Palladium Nanoparticles. J Am Chem Soc 123(28): 6840-6846.

15. HJ Xu, YQ Zhao, XF Zhou (2011) Palladium-Catalyzed Heck Reaction of Aryl Chlorides under Mild Conditions Promoted by Organic Ionic Bases. J Org Chem 76(19): 8036-8041.

16.Q Liang, P Xing, Z Huang, J Dong, KB Sharpless, et al. (2015) Palladium-Catalyzed, Ligand-Free Suzuki Reaction in Water Using Aryl Fluorosulfates. Org Lett 17(8): 1942-1945.

17. PK Khanna, BG Bharate, Priyesh More, N Koteswara Rao, VN Singh, et al. (2009) Synth React Inorg Met-Org. Chem 37(7): 367-372.

18. S Moisan, V Martinez, P Weisbecker, F Cansell, S Mecking, et al. (2007) J Am Chem Soc 129: 10602-10606.

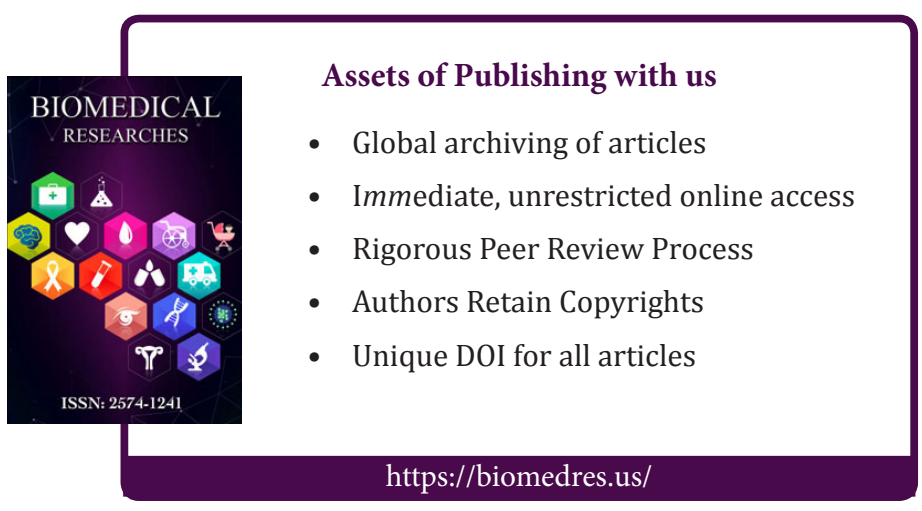

